\title{
Numerical Simulations of the FIRE-II Convective and Radiative Heating Rates
}

\author{
Leonardo C. Scalabrin* and Iain D. Boyd ${ }^{\dagger}$ \\ Department of Aerospace Engineering, University of Michigan, Ann Arbor, MI, 48109, USA
}

\begin{abstract}
Numerical simulations of axisymmetric flows over the FIRE-II spacecraft at reentry conditions using a Navier-Stokes solver being developed at the University of Michigan are presented. The finite-volume method is used to solve the set of differential equations. The code has the capability to handle any mix of hexahedra, tetrahedra, prisms and pyramids in $3 \mathrm{D}$ or triangles and quadrilaterals in $2 \mathrm{D}$. Numerical fluxes between the cells are discretized using a modified Steger-Warming Flux Vector Splitting which has low dissipation and is appropriate to calculate boundary layers. A point or line implicit method is used to perform the time integration. The computed convective heating rates are in good agreement to other numerical results and are consistent with total heat transfer flight measurements. The radiative heating rates, computed using NASA's NEQAIR code, are also in good agreement with results obtained previously.
\end{abstract}

\section{Introduction}

$\mathrm{T}_{\mathrm{T}}^{\mathrm{o} w \mathrm{w}}$ ODAY, numerical simulation of reentry flows is an integral part in the design process of reentry vehicles. ${ }^{1}$ The simulation of weakly ionized gases over reentry vehicles has been performed successfully for a large number of spacecraft, including RAM-C, ${ }^{2}$ FIRE, ${ }^{3,4}$ Apollo Command Module, ${ }^{5}$ Huygens ${ }^{6}$ and Mars Science Laboratory. ${ }^{7}$ Computational aerothermodynamics is being routinely used at NASA in the design of the next manned spacecraft, Orion.

One of the most important parameters for design and one of the most difficult to calculate numerically is the heat transfer to the spacecraft. The heat transfer is composed of two parts: convective and radiative heating. For the stagnation point of a blunt body, the convective heat transfer rate is roughly proportional ${ }^{8}$ to $\rho^{0.5} u^{3.0} / R_{N}^{0.5}$ where $\rho$ is the freestream density, $u$ is the freestream speed and $R_{N}$ is the nose radius. The radiative heat transfer is roughly proportional ${ }^{8}$ to $\rho^{1.6} u^{8.5} R_{N}$. The convective heating is usually the largest component but the previous expressions show that the radiative heating component becomes increasingly important as the size of the spacecraft grows. Orion diameter is projected to be around 5 meters making it larger than its predecessor Apollo, which measured 4.3 meters in diameter. Because of the increased diameter, there is a lot of interest in the radiative heating component. The FIRE-II flight experiment from the 1960's still is one of the best set of data available for radiative and total heating measurements. It is commonly used as a validation test case for aerothermodynamics codes. ${ }^{3,4,9}$

In one of these validation studies, ${ }^{9}$ it was observed that two independent NASA codes, DPLR ${ }^{10}$ (developed at NASA Ames) and LAURA ${ }^{11}$ (developed at NASA Langley), showed good agreement for computed convective heating rates with the difference staying below $7 \%$ for 11 -species air model simulations. That work also showed larger differences in the computed radiative heating rate, on the order of 17 to $25 \%$. The physical and numerical modeling used in these codes is complex and it is not known which model may be the cause for such differences. A sensitivity analysis may be performed to obtain information on the impact of modeling choices on the results and to explain the differences.

This paper presents recent developments of a numerical code able to simulate hypersonic flows in thermochemical nonequilibrium. The current code is an extension of an earlier version of the code $\mathrm{e}^{12,13}$ modified to handle weakly ionized gases at speeds higher than $10 \mathrm{~km} / \mathrm{s}$ when some of the simpler physical models are no longer adequate. This paper shows comparisons of convective and radiative heating obtained using the

\footnotetext{
* Graduate Student, Student Member AIAA.

${ }^{\dagger}$ Professor, Associate Fellow AIAA
} 
present code against established NASA codes as part of its validation effort. In addition, sensitivity analysis of the radiative heating to some of the thermal non-equilibrium models is also performed to investigate possible reasons for poorer agreement in the radiation results.

\section{Physical Modeling}

The flow is modeled assuming that the continuum approximation is valid and that the fluid can be weakly ionized. Furthermore, it is assumed that the rotational and translational energy modes of all species can be described by a single temperature $T$ and that the vibrational energy mode of all species and the electron energy can be described by a single temperature $T_{v}$. With those approximations, the conservation equations for the two-dimensional system can be written as $^{2,11,14,15}$

$$
\frac{\partial Q}{\partial t}+\frac{\partial\left(F-F_{v}\right)}{\partial x}+\frac{\partial\left(G-G_{v}\right)}{\partial y}=S_{c v}
$$

where

$$
Q=\left\{\begin{array}{lllllll}
\rho_{1} & \cdots & \rho_{n s} & \rho u & \rho v & E & E_{v}
\end{array}\right\}^{T}
$$

is the vector of conserved variables. In these expressions, $\rho_{1} \cdots \rho_{n s}$ are the species mass densities, $u$ and $v$ are the bulk velocity components, $E$ is the total energy per unit volume of mixture given by

$$
E=\sum_{s \neq e} \rho_{s} C v_{s} T+\frac{1}{2} \rho\left(u^{2}+v^{2}\right)+\sum_{s \neq e} \rho_{s} h_{s}^{o}+E_{v} \quad,
$$

where $C v_{s}$ is the species specific heat at constant volume, $h_{s}^{o}$ is the species heat of formation at $0 \mathrm{~K}$ and

$$
E_{v}=\sum_{s \neq e} \rho_{s} e_{v, s}+\sum_{s \neq e} \rho_{s} e_{e l, s}+\rho_{e} e_{e}
$$

is the vibrational energy per unit volume. In the last equation, the species vibrational energy per unit mass, $e_{v, s}$, is modeled using a harmonic oscillator for which

$$
e_{v, s}=\frac{R u}{M_{s}} \frac{\theta_{v, s}}{\exp \left(\theta_{v, s} / T_{v}\right)-1},
$$

where $R u$ is the universal gas constant, $M_{s}$ is the species molecular weight and $\theta_{v, s}$ is the harmonic oscillator characteristic temperature. The species electronic energy, $e_{e l, s}$ is modeled by

$$
e_{e l, s}=\frac{R u}{M_{s}} \frac{\sum_{i=1}^{\infty} g_{i, s} \theta_{e l, i, s} \exp \left(-\theta_{e l, i, s} / T_{v}\right)}{\sum_{i=0}^{\infty} g_{i, s} \exp \left(-\theta_{e l, i, s} / T_{v}\right)},
$$

where $\theta_{e l, i, s}$ and $g_{i, s}$ are the characteristic electronic temperature and the degeneracy of the i-th energy level, respectively. The electron energy, $e_{e}$, is given by

$$
e_{e}=C v_{e} T_{v}
$$

The inviscid and viscous flux vector components in the $x$ direction are given by

$$
F=\left\{\begin{array}{c}
\rho_{1} u \\
\vdots \\
\rho_{n s} u \\
\rho u^{2}+p \\
\rho u v \\
(E+p) u \\
E_{v} u
\end{array}\right\} \quad \text { and } \quad F_{v}=\left\{\begin{array}{c}
-J_{x, 1} \\
\vdots \\
-J_{x, n s} \\
\tau_{x x} \\
\tau_{x y} \\
\tau_{x x} u+\tau_{x y} v-\left(q_{t, x}+q_{r, x}+q_{v, x}\right)-\sum\left(J_{x, s} h_{s}\right) \\
-q_{v, x}-\sum\left(J_{x, s} e_{v, s}\right)
\end{array}\right\}
$$

where $p$ is the pressure, $\tau_{i j}$ are the viscous stress components and $q_{t, i}, q_{r, i}$ and $q_{v, i}$ are the translational, rotational and vibrational heat fluxes in the $i$-th direction. Moreover, $h_{s}$ is the species enthalpy and $J_{x, s}$ is the species diffusion flux in the $x$-direction. The flux vectors in the other directions are similar. 
The pressure is calculated assuming that each species can be modeled using a perfect gas relation and Dalton's law of partial pressures so that

$$
p=\sum_{s \neq e} \rho_{s} \frac{R u}{M_{s}} T+\rho_{e} \frac{R u}{M_{e}} T_{v} .
$$

The species enthalpies are given by

$$
h_{s}= \begin{cases}\left(C v_{t, s}+C v_{r, s}+R_{s}\right) T+h_{s}^{o}+e_{v, s}+e_{e l, s} & \text { for molecules } \\ \left(C v_{t, s}+R_{s}\right) T+h_{s}^{o}+e_{e l, s} & \text { for atoms } \\ \left(C v_{t, e}+R_{e}\right) T_{v} & \text { for electrons. }\end{cases}
$$

where $C v_{t, s}$ and $C v_{r, s}$ are the species translational and rotational specific heats at constant volume.

The viscous stresses are modeled assuming a Newtonian fluid for which, using Stokes' hypothesis,

$$
\tau_{i j}=\mu\left(\frac{\partial u_{j}}{\partial x_{i}}+\frac{\partial u_{i}}{\partial x_{j}}\right)+\lambda \nabla \vec{u} \delta_{i j} \quad, \quad \lambda=-\frac{2}{3} \mu \quad,
$$

where $\mu$ is the mixture coefficient of viscosity. The heat fluxes are modeled according to Fourier's law as

$$
\vec{q}_{t, r, v}=-\kappa_{t, r, v} \nabla T_{t, r, v}
$$

where $\kappa_{t, r, v}$ are the mixture thermal conductivity for each energy mode. The species mass diffusion fluxes are modeled using Fick's law modified to enforce that the sum of the diffusion fluxes is zero

$$
\vec{J}_{s \neq e}=\vec{I}_{s}-\sum_{r \neq e} \vec{I}_{r}
$$

where

$$
\vec{I}_{s}=-\rho D_{s} \nabla Y_{s} \quad,
$$

where $D_{s}$ is the species diffusion coefficients and $Y_{s}$ is the species mass fraction. The electron diffusion flux is not included in Eq. 13 because its value is assumed small. Instead, the diffusion flux of electrons is calculated using ambipolar diffusion to guarantee the charge neutrality in the flowfield

$$
\overrightarrow{J_{e}}=M_{e} \sum_{s \neq e} \frac{\vec{J}_{s} C_{s}}{M_{s}}
$$

where $M_{e}$ is the electron molecular weight and $C_{s}$ is the species charge.

Two different models to calculate mixture transport properties are available. One uses Wilke's semiempirical mixing rule ${ }^{16}$ with species viscosities calculated using Blottner's model ${ }^{17}$ and species thermal conductivities determined using Eucken's relation. ${ }^{18}$ The other option uses Gupta's mixing rule ${ }^{19}$ with species viscosities and thermal conductivities calculated using non-coulombic/coulombic collision cross section data compiled by Wright. ${ }^{20}$ The diffusion coefficients for all species can be assumed equal as a simple way to ensure that the sum of diffusion fluxes is equal to zero ${ }^{21}$ or they can be calculated using Gupta's mixing rule ${ }^{19}$ and the collision cross section data. ${ }^{20}$

The source term, $S_{c, v}$, is given by

$$
S_{c, v}=\left\{\begin{array}{ccccccc}
\dot{w}_{1} & \cdots & \dot{w}_{n s} & 0 & 0 & 0 & \dot{w}_{v}
\end{array}\right\}^{T}
$$

where $\dot{w}_{1} \cdots \dot{w}_{n s}$ are the species mass production rates by chemical reactions and $\dot{w}_{v}$ is the vibrational energy source term. The species mass production rates are modeled using a standard finite-rate chemistry model for reacting air in conjunction with Park's two-temperature model ${ }^{15}$ to account for thermal nonequilibrium effects on the reaction rates. The forward reaction rates used in this work are taken from Ref. 22 or 23 . The backward reaction rates are calculated using curve fits for equilibrium constants taken from Ref. 15 or 23 or using Gibb's free energy as.

$$
K_{r}=\left(\frac{p_{o}}{R T}\right)^{\nu_{r}} \exp \left\{-\sum_{s}\left(\nu_{s, r}^{\prime \prime}-\nu_{s, r}^{\prime}\right)\left[\left(\frac{\hat{h}_{s}}{R T}-\frac{\hat{s}_{s}}{R}\right)\right]\right\},
$$


where $p_{o}$ is a reference pressure set to 1 bar and

$$
\nu_{r}=\sum_{s}\left(\nu_{s, r}^{\prime \prime}-\nu_{s, r}^{\prime}\right)
$$

The vibrational energy source term is given by

$$
\dot{w}_{v}=-p_{e} \nabla \cdot \vec{u}+\sum \dot{w}_{s}\left(D_{s}^{\prime}+e_{e l, s}\right)+S_{t-v}+S_{h-e} \quad,
$$

where $p_{e} \nabla \cdot \vec{u}$ is an approximation to the work done on electrons by the electric field induced by the electron pressure gradient ${ }^{2,11}$ and $D_{s}^{\prime}$ is the vibrational energy of molecules added or removed by recombination or dissociation respectively, which can be modeled using a preferential or non-preferential model. In the non-preferential model,

$$
D_{s}^{\prime}=e_{v, s}
$$

and in the preferential model,

$$
D_{s}^{\prime}=\alpha D_{s} \quad
$$

where $\alpha$ is typically set to 0.3 and $D_{s}$ is the dissociation potential of the molecule. The energy exchange rate between the translation-rotational and the vibrational energy modes, $S_{t-v}$, is modeled using the LandauTeller model ${ }^{18}$ and Millikan and White's correlation ${ }^{24}$ with exceptions for some pairs of interactions. ${ }^{22} \mathrm{~A}$ high temperature correction for limiting equivalent collision cross sections is also employed. ${ }^{25}$ The energy transfer rate between heavy particles and electrons, $S_{h-e}$, is given by

$$
S_{h-e}=3 R u \rho_{e}\left(T-T_{v}\right) \sqrt{\frac{8 R u T_{v}}{\pi M_{e}}} \sum_{r \neq e} \frac{\rho_{r} N_{a}}{M_{r}^{2}} \sigma_{e r},
$$

where $\sigma_{e r}$ are the collision cross sections between electrons and heavy particles. ${ }^{2,14}$

\section{Numerical Modeling}

The finite-volume method applied to unstructured grids is used to solve the set of differential equations. ${ }^{26}$ The present code, "Le" Michigan Aerothermodynamics Navier-Stokes Solver (LEMANS) is capable of simulating 2D/Axisymmetric flows using any mix of quadrilaterals and triangles, and 3D flows using any mix of hexahedra, tetrahedra, prisms and pyramids. For axisymmetric simulations, the source terms that arise from the finite-volume integration of the system of equations are taken from Ref. 27. The inviscid fluxes across cell faces are discretized using a modified form of the Steger-Warming Flux Vector Splitting (FVS) scheme $^{28}$ which is less dissipative and yields better results in boundary layers. The method switches to the original Steger-Warming FVS scheme ${ }^{29,30}$ at shock waves by using a pressure switch. ${ }^{31}$ The viscous terms are calculated using the values of properties at the cell centers and at the nodes. ${ }^{32}$ The property values at the nodes are calculated using a simple average of the values of the cells that share that node. Use of this method increases the stencil used in the derivative calculations in order to avoid loss of accuracy when using unstructured meshes. ${ }^{32}$ Time integration is performed using a point implicit or a line implicit method. ${ }^{10,33,34}$ The code is parallelized using MPI to allow the simulation of large problems. The computational grid partitioning is performed using METIS.

LEMANS has been successfully used to simulate a variety of hypersonic flows ${ }^{13}$ at Mach numbers ranging from 10 to 23. The physical modeling described in this paper is included in the code to allow accurate simulations of more severe conditions such as the FIRE-II reentry for which the Mach number can get as high as 40. During the implementation of these models in the code, it was observed that a diffusion model that accounts for different diffusion coefficients for each species and the inclusion of excited electronic states are necessary to obtain good results.

\section{Results}

The FIRE-II flight experiment was performed in 1965 in order to validate the aerothermodynamic models used to design the Apollo thermal protection system. The flight experiment measured the total and radiative heating rates to the spacecraft. The FIRE-II thermal protection system used three non-ablating heat shields 
made of beryllium metal and asbestos impregnated phenolic resin heat shields in between them. The heat shields were ejected at prescribed times so that a new beryllium heat shield was exposed during each measurement period. Each measurements period ended when the corresponding beryllium heat shield started to ablate. The three freestream conditions in this work are the same as in Ref. ${ }^{9}$ They are chosen such that each one corresponds to a different beryllium heat shield. The conditions and basic geometry information are tabulated in Table 1 where $t$ is the flight time, $H$ is the altitude, $R_{n}$ is the nose radius, $R_{c}$ is the shoulder radius and $R_{b}$ is the base radius. All conditions satisfy the limit for a continuum approximation $-K n<0.01$ and all of them are in the laminar regime.

Table 1. Freestream Conditions.

\begin{tabular}{|ccccccccc|}
\hline$t$ & $H$ & $u_{\infty}$ & $\rho$ & $T_{\infty}$ & $T_{w}$ & $R_{n}$ & $R_{c}$ & $R_{b}$ \\
$(s)$ & $(\mathrm{km})$ & $(\mathrm{m} / \mathrm{s})$ & $\left(\mathrm{kg} / \mathrm{m}^{3}\right)$ & $(K)$ & $(K)$ & $(m)$ & $(\mathrm{m})$ & $(\mathrm{m})$ \\
\hline 1636 & 71.04 & $1.131 \times 10^{4}$ & $8.57 \times 10^{-5}$ & 210 & 810 & $9.347 \times 10^{-1}$ & $1.02 \times 10^{-2}$ & $3.3575 \times 10^{-1}$ \\
1643 & 53.04 & $1.048 \times 10^{4}$ & $7.80 \times 10^{-4}$ & 276 & 640 & $8.052 \times 10^{-1}$ & $3.56 \times 10^{-2}$ & $3.1495 \times 10^{-1}$ \\
1651 & 37.19 & $6.19 \times 10^{3}$ & $6.05 \times 10^{-3}$ & 253 & 1060 & $7.021 \times 10^{-1}$ & $0.61 \times 10^{-2}$ & $2.9395 \times 10^{-1}$ \\
\hline
\end{tabular}

\section{A. Convective Heating}

A total of 12 simulations are performed using LEMANS and compared to DPLR and LAURA. The simulations used 5 and 11-species air models and catalytic and supercatalytic wall boundary conditions for the 3 different trajectory times listed in Table 1 . The results for convective heating are presented in Table 2, where the data for DPLR and LAURA are obtained from Ref. 9. The results for LEMANS lie in between DPLR and LAURA for all conditions. One of the most interesting cases is the 1636, 11-species case which given the high speed and low density, can highlight the differences between the physical modeling in the codes. For that particular case, the heat transfer calculated using LEMANS is very close to the one calculated by DPLR. That is not too surprising since much of the modeling inside LEMANS and DPLR is the same, including inviscid fluxes, collision integrals and chemistry rates. Overall, the table shows that the difference between the 3 codes for 11-species cases is at most 7\%, a very good comparison given all the physical and numerical modeling involved. The results are consistent with the total heating rates measured in flight for the three conditions that are 290,1025, and $390 \mathrm{~W} / \mathrm{cm}^{2}$ for the 1636, 1643 and 1651 seconds flight times respectively.

Table 2. Convective Heating

\begin{tabular}{|c|c|c|c|c|c|c|}
\hline $\begin{array}{c}\text { Time } \\
(\mathrm{s})\end{array}$ & $\begin{array}{c}\text { Air Model } \\
\text { (No. of species) }\end{array}$ & $\begin{array}{c}\text { Wall Catalicity } \\
(\text { Yes/No })\end{array}$ & $\begin{array}{c}\text { DPLR } \\
\left(W / \mathrm{cm}^{2}\right)\end{array}$ & $\begin{array}{l}\text { LAURA } \\
\left(W / \mathrm{cm}^{2}\right)\end{array}$ & $\begin{array}{l}\text { LEMANS } \\
\left(\mathrm{W} / \mathrm{cm}^{2}\right)\end{array}$ & $\begin{array}{c}\text { Difference } \\
(\%)\end{array}$ \\
\hline \multirow{4}{*}{1636} & \multirow{2}{*}{11} & No & 183 & 191 & 185 & 4 \\
\hline & & Yes & 301 & 307 & 307 & 2 \\
\hline & \multirow{2}{*}{5} & No & 226 & 204 & 210 & -10 \\
\hline & & Yes & 365 & 340 & 346 & -7 \\
\hline \multirow{4}{*}{1643} & \multirow{2}{*}{11} & No & 769 & 753 & 753 & -2 \\
\hline & & Yes & 805 & 802 & 810 & -1 \\
\hline & \multirow{2}{*}{5} & No & 823 & 773 & 799 & -6 \\
\hline & & Yes & 904 & 839 & 860 & -7 \\
\hline \multirow{4}{*}{1651} & \multirow{2}{*}{11} & No & 478 & 449 & 473 & -6 \\
\hline & & Yes & 511 & 477 & 503 & -7 \\
\hline & \multirow{2}{*}{5} & No & 477 & 451 & 473 & -5 \\
\hline & & Yes & 511 & 479 & 505 & -6 \\
\hline
\end{tabular}

In order to illustrate the results, the 1636 seconds , 11-species non-catalytic wall case is discussed in more 
details. The mesh used in this calculation is presented in Fig. 1. It has 18, 432 cells with 144 in the normal direction and 128 along the body. The spacing at the wall is around $2 \times 10^{-6}$ meters.

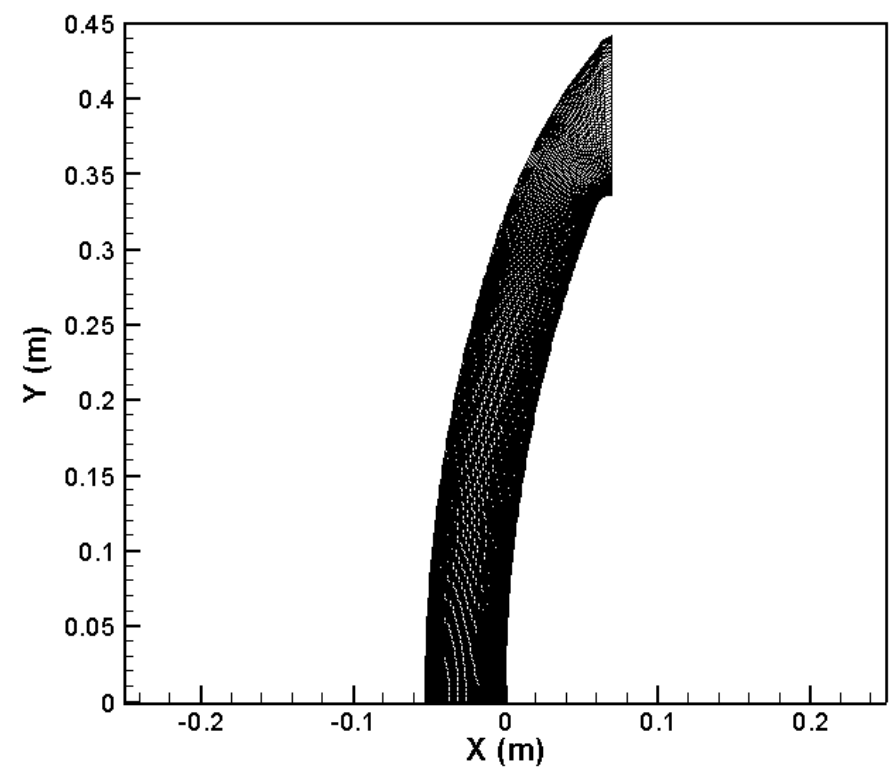

Figure 1. Mesh used by DPLR and LEMANS for the 1636 seconds, 11-species cases.

A general overview of the flowfield is presented in Fig. 2, which shows contours of translational temperature and pressure in the flowfield. The maximum temperature in the flowfield is around $30,000 \mathrm{~K}$ occurring immediately after the shock wave. Due to dissociation and ionization reactions, the temperature decreases to around 10,000 $\mathrm{K}$ in the shock layer. The pressure contours show the expected blunt body behavior with a high pressure at the stagnation region reaching values close to 10,000 $\mathrm{Pa}$ and an expansion around the shoulder of the spacecraft. The 2 nd order scheme employed in the numerical calculation makes the contour lines very sharp.

The high energy of the flow causes high rates of chemical reactions after the shock wave leading to a drop in temperature after the shock. Figure 3 shows the species mass fractions along the stagnation streamline. Closer to the shock wave, the ions with larger mass fractions are $\mathrm{N}_{2}^{+}, \mathrm{O}_{2}^{+}$and $\mathrm{NO}^{+}$which are formed through associative ionization reactions. The atomic species $N, O$ and their ions $N^{+}$and $\mathrm{O}^{+}$are the species with larger mass fractions in the shock layer followed by $N_{2}$. Inside the boundary layer, the decrease in temperature causes increased rates of recombination reactions.

Figure 4 shows a comparison of heat transfer profiles calculated by LEMANS, DPLR and LAURA. The agreement between LEMANS and DPLR is remarkable. Both codes show a small dip in the profile close to the stagnation point, which could be attributed to a "Carbuncle" like behavior. It is well known in the CFD community that grid alignment to the shock wave and the inviscid fluxes play important roles in the appearance of "carbuncles". Both LEMANS and DPLR use the same grid and same inviscid numerical fluxes for these calculations which may explain why both codes present a similar behavior close to the stagnation point.

A comparison between the translational temperature profiles along the stagnation streamline calculated by the three codes is presented in Fig. 5. It can be observed that the three codes present good agreement in the equilibrium temperature after the shock and inside the boundary layer. The codes show distinct behavior at the shock wave. LAURA predicts a broader shock and the largest peak translational temperature while DPLR presents a thinner shock and the smallest peak in translational temperature. LEMANS stands somewhere in between both codes. LAURA broader shock comes from a coarser grid that has 6,400 cells with 80 in the normal direction and 80 along the body. This grid, shown in Fig. 6, is clearly coarser close to the freestream boundary. While LEMANS and DPLR used the same mesh, LEMANS present a larger shock stand off distance. That is caused by the modeling of electron impact ionization reactions as discussed in 


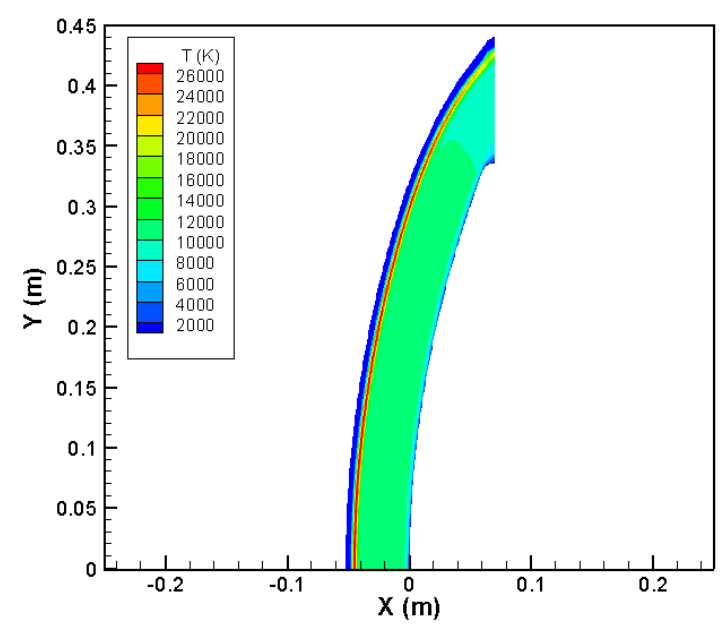

(a) Temperature

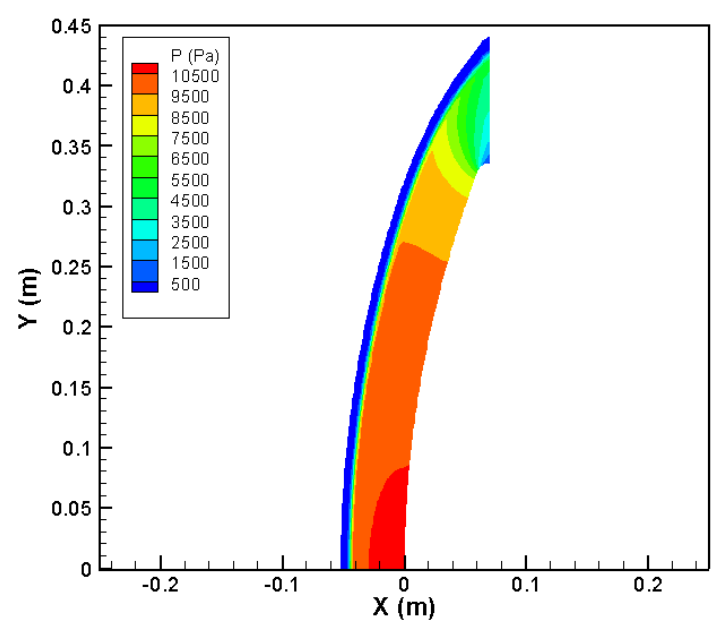

(b) Pressure

Figure 2. Flowfield around the FIRE-II spacecraft for the 1636 seconds, 11-species, non-catalytic wall case.

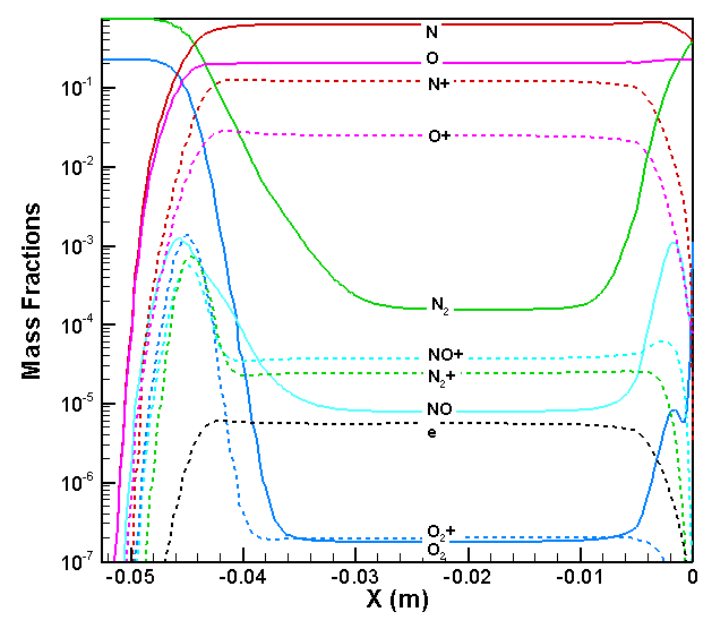

Figure 3. Species mass fractions along the stagnation line for the 1636 seconds, 11-species, non-catalytic wall case. 


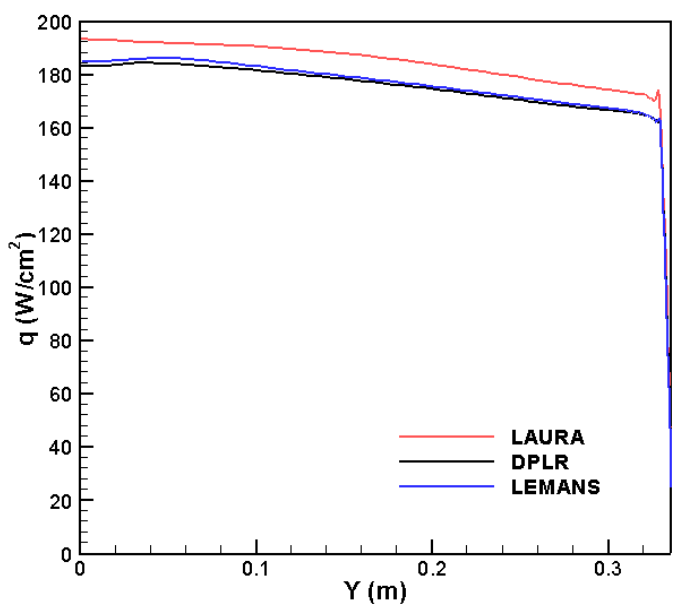

Figure 4. Convective heat transfer along the wall for the 1636 seconds, 11-species, non-catalytic wall case.

the next section.

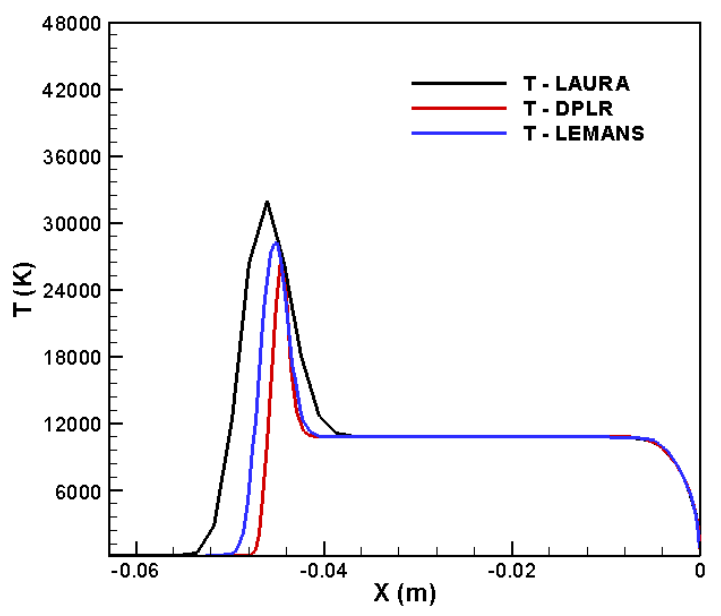

Figure 5. Translational temperature profiles along the stagnation line for the 1636 seconds, 11-species, noncatalytic wall case.

Figure 7 presents comparisons of the vibrational temperature profiles along the stagnation line calculated by the three codes. While the codes agree on the equilibrium value after the shock, the profiles are different at the shock wave and inside the boundary layer. LAURA and LEMANS predict a somewhat higher value of vibrational temperature inside the boundary layer when compared to DPLR. In addition, LAURA and LEMANS profiles present a peak in vibrational temperature at the shock while DPLR profile does not. LAURA and LEMANS couple the electron and electronic energy with the vibrational energy while DPLR couples the electron and electronic energy with the translational mode. The peak in vibrational temperature for LAURA and LEMANS is caused by the increase of electron and electronic energy close to the shock wave. 


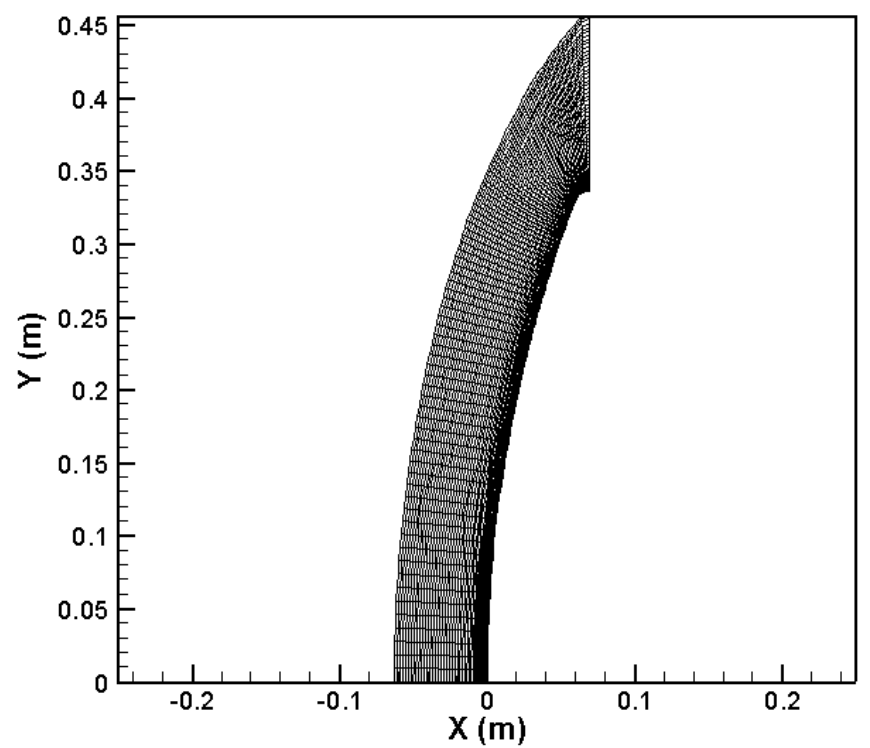

Figure 6. Mesh used by LAURA for the 1636 seconds, 11-species cases.

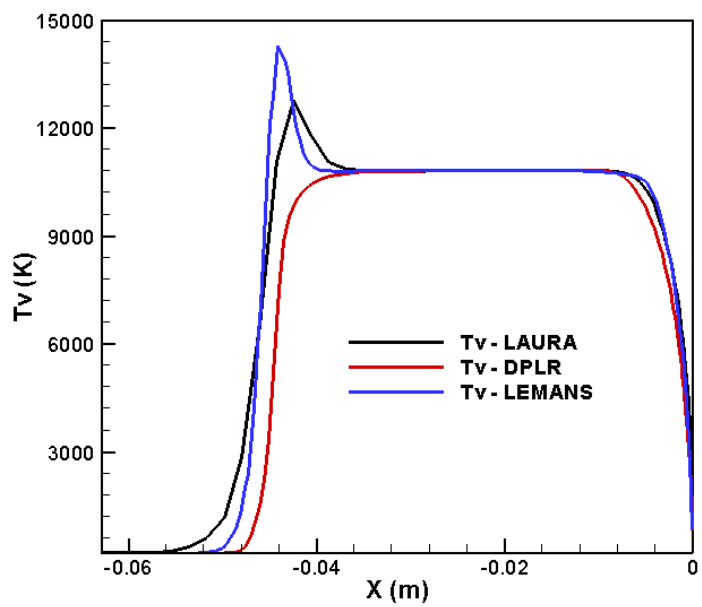

Figure 7. Vibrational temperature profiles along the stagnation line for the 1636 seconds, 11-species, noncatalytic wall case. 


\section{B. Radiative Heating}

The radiative heating calculations in this study are performed using NEQAIR. ${ }^{35}$ NEQAIR is run using the Quasi-Steady State (QSS) assumption in which excitation rates are computed using the electron temperature. NEQAIR requires the translational, rotational, vibrational and electron temperatures and species number densities along a line of sight in the flow field to calculate the radiation. In this work, only the stagnation line is used. The 1636 seconds, 11-species case is used for comparing results obtained using the three different CFD codes. Results for DPLR and LAURA are from Ref. 9. Table 3 shows the results from all codes for catalytic and non-catalytic wall conditions including the percentage difference in relation to LAURA results. LEMANS results are again in between those calculated by DPLR and LAURA for the VUV radiation range but about $10 \%$ lower for the long wavelength region of the spectrum for which flight data is available. For $200 \mathrm{~nm}<\lambda<4 \mu \mathrm{m}$ the flight data radiative heating rate is $28.5 \mathrm{~W} / \mathrm{cm}^{2}$. The agreement for the radiative heating between the three codes is not as good as for the convective heating. Most of the radiation to the spacecraft during a reentry in the earth atmosphere is related to the electron temperature and the number densities of electrons, atomic nitrogen and atomic oxygen. The electron temperature for LAURA and LEMANS is assumed to be in equilibrium with the vibrational temperature while in DPLR the electron temperature is assumed in equilibrium with the translational temperature. All codes assume that the rotational temperature is equilibrated with the translational temperature.

Figure 8 compares the electron number density profiles calculated by the three codes. It is clear from this figure that DPLR and LEMANS profiles present a peak close to the shock while LAURA profile does not. The maximum electron number density calculated by DPLR is much larger than the calculated by LAURA while LEMANS lies somewhere in between. DPLR and LAURA profiles agree very well inside the boundary layer while LEMANS profile differs from both in that region. The disagreement comes from extra numerical dissipation in the inviscid fluxes which is switched off closer to the wall in LEMANS than it is in LAURA and DPLR. The extra numerical dissipation is obtained by modifying the inviscid fluxes eigenvalues. ${ }^{30,31}$

The profiles for atomic nitrogen are presented in Fig. 9. The difference between the three codes is much smaller in this case. The difference between LEMANS and the other codes in the boundary layer is due to the extra numerical dissipation as mentioned earlier. At the shock wave, LEMANS and DPLR profiles seem to be in better agreement. LAURA profile close to the shock is somewhat more diffused. The behavior for atomic oxygen is similar. The number density profiles for this species are presented in Fig. 10. LEMANS profile disagrees from DPLR and LAURA in the boundary layer due to extra numerical dissipation. LAURA profile is also more diffused at the shock wave.

Table 3. Stagnation point radiative heating calculations using different codes.

\begin{tabular}{ccccc}
\hline & \multicolumn{4}{c}{ Heating rate $\left(W / \mathrm{cm}^{2}\right)$} \\
Code & \multicolumn{2}{c}{ Non-Catalytic } & \multicolumn{2}{c}{ Catalytic } \\
& $\lambda<200 \mathrm{~nm}$ & $\lambda \geq 200 \mathrm{~nm}$ & $\lambda<200 \mathrm{~nm}$ & $\lambda \geq 200 \mathrm{~nm}$ \\
& $(\mathrm{VUV})$ & $(\mathrm{UV}+\mathrm{Vis}+\mathrm{IR})$ & $(\mathrm{VUV})$ & $(\mathrm{UV}+\mathrm{Vis}+\mathrm{IR})$ \\
\hline LAURA & 42.06 & 28.03 & 39.24 & 28.00 \\
Diff. & - & - & - & - \\
\hline DPLR & 49.33 & 26.02 & 49.21 & 25.38 \\
Diff. & $17 \%$ & $-7 \%$ & $25 \%$ & $-9 \%$ \\
\hline LEMANS & 44.26 & 24.79 & 44.48 & 23.98 \\
Diff. & $5 \%$ & $-12 \%$ & $13 \%$ & $-14 \%$ \\
\hline
\end{tabular}

Given the differences in the physical modeling employed by the codes, a sensitivity analysis is performed to understand the reasons for the differences in the stagnation line property profiles. The sensitivity analysis focuses on the thermal non-equilibrium model because the flow is in thermo-chemical equilibrium in the shock layer. The thermal non-equilibrium model impacts the profiles close to shock wave which is the region where the profiles show larger differences as seen in Figs. 5 to 10. Table 4 shows the impact of changes in some aspects of the thermochemistry modeling on the calculated radiation, including the percentage difference in relation to LEMANS baseline result.

The first change is to simulate a thermal-equilibrium flow. This is achieved by using a constant vibrational- 


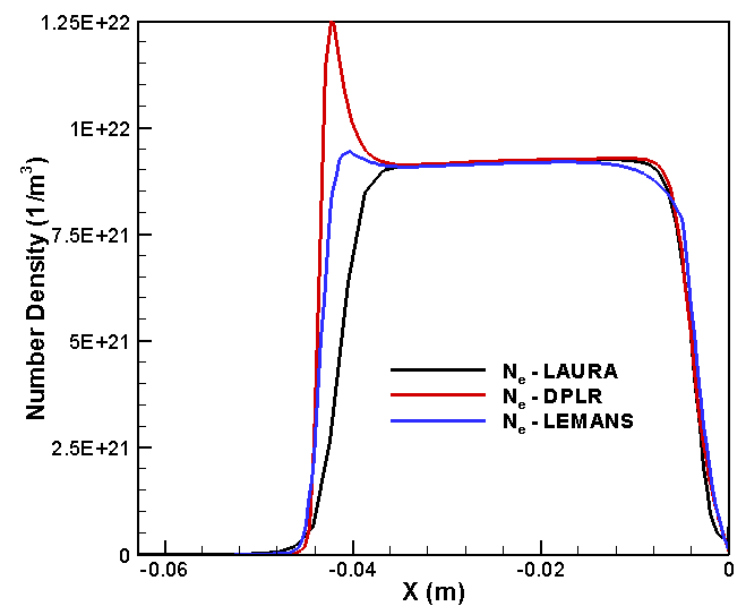

Figure 8. Electron number density along the stagnation line for the 1636 seconds, 11-species, non-catalytic wall case calculated using different codes.

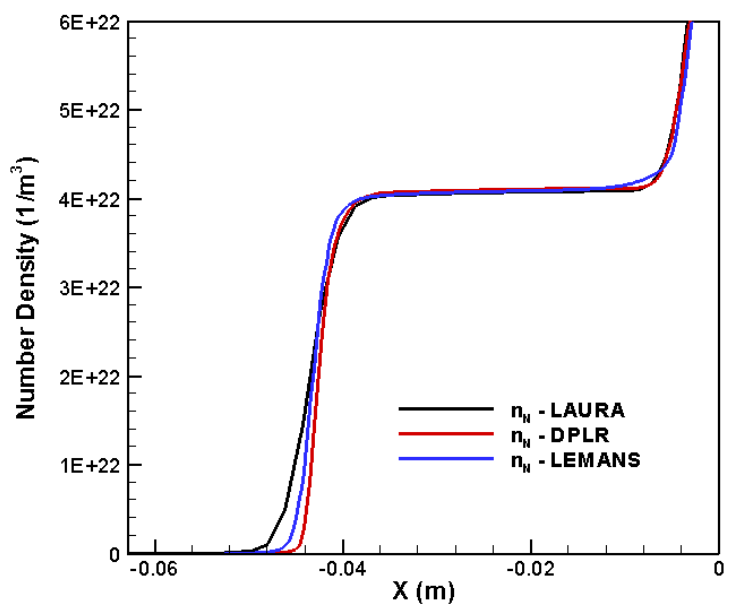

Figure 9. Atomic nitrogen number density along the stagnation line for the 1636 seconds, 11-species, noncatalytic wall case calculated using different codes. 


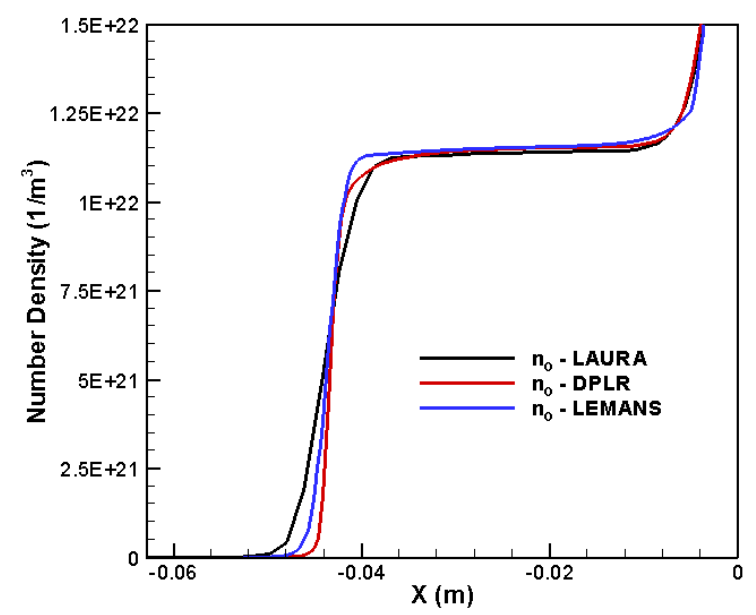

Figure 10. Atomic oxygen number density along the stagnation line for the 1636 seconds, 11-species, noncatalytic wall case calculated using different codes.

Table 4. Stagnation point radiative heating sensitivity analysis for Non-Catalytic wall.

\begin{tabular}{ccc}
\hline & \multicolumn{2}{c}{ Heating rate $\left(W / \mathrm{cm}^{2}\right)$} \\
Parameter & $\lambda<200 \mathrm{~nm}$ & $\lambda \geq 200 \mathrm{~nm}$ \\
& $(\mathrm{VUV})$ & $(\mathrm{UV}+\mathrm{Vis}+\mathrm{IR})$ \\
\hline Baseline & 44.26 & 24.79 \\
Diff. & - & - \\
\hline Thermal-Equilibrium & 43.48 & 24.34 \\
Diff. & $-1.8 \%$ & $-1.8 \%$ \\
\hline Impact Ionization & 44.55 & 24.41 \\
Diff. & $0.7 \%$ & $-1.5 \%$ \\
\hline Constant $\tau_{v}=1 \times 10^{-5}$ & 42.43 & 24.41 \\
Diff. & $-4.1 \%$ & $-1.5 \%$ \\
\hline Pref. Diss. Model & 44.15 & 24.78 \\
Diff. & $-0.2 \%$ & $0.0 \%$ \\
\hline$T^{0.7} T_{v}^{0.3}$ & 44.41 & 24.82 \\
Diff. & $0.3 \%$ & $0.1 \%$ \\
\hline Laura's Chemistry Set & 43.46 & 25.38 \\
Diff. & $-1.8 \%$ & $2.4 \%$ \\
\hline Laura's Grid & 42.67 & 24.01 \\
Diff. & $-3.6 \%$ & $-3.1 \%$ \\
\hline & &
\end{tabular}


electron-electronic relaxation time of the order $1 \times 10^{-13}$ seconds. The equilibrium assumption drastically reduced the maximum translational temperature in the flowfield and the shock stand-off distance, as can be observed in Fig. 11. The reduction in temperature is due to the immediate energy transfer to the other energy modes. The smaller temperature makes the fluid denser, thus reducing the shock stand-off distance. The vibrational temperature is a little larger in the thermal equilibrium simulation, as can be observed in Fig. 12, but it is in good agreement with the baseline solution. The thermal equilibrium calculation does not cause significant differences in the number densities of electrons, nitrogen and oxygen along the stagnation line as shown in Figs. 13, 14 and 15. Because of such small differences in vibrational temperature and the number densities, the difference in the calculated radiation is small.

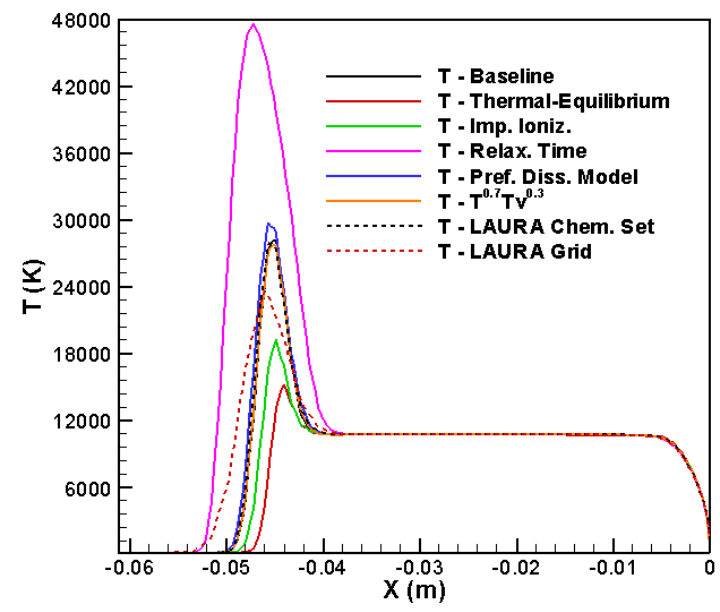

Figure 11. Translational temperature profiles along the stagnation line for the 1636 seconds, 11-species, noncatalytic wall case.

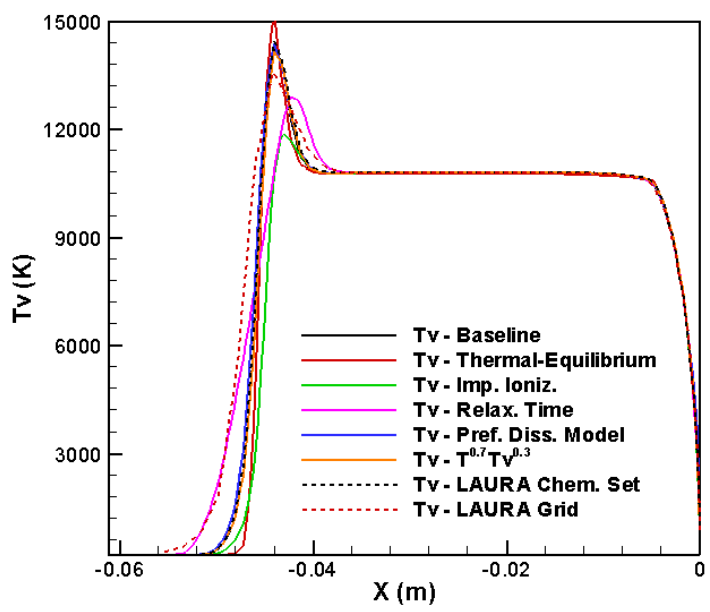

Figure 12. Vibrational temperature profiles along the stagnation line for the 1636 seconds, 11-species, noncatalytic wall case.

The second modification changes the model for impact ionization reactions. In LAURA those reaction rates are function of the vibrational-electron-electronic temperature only while in DPLR they are function of the translational temperature only. This change reduces the translational temperature significantly as seen in Fig. 11, because it causes those endothermic reactions to occur close to the shock wave. As shown in Fig. 5, the smaller temperature also reduces the shock stand-off distance, yielding good agreement to the 


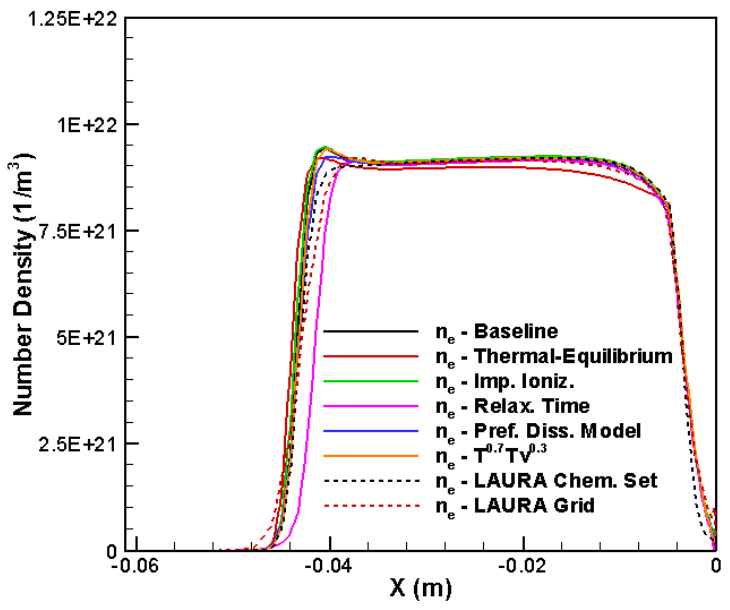

Figure 13. Electron number density along the stagnation line for the 1636 seconds, 11-species, non-catalytic wall case calculated using different thermal non-equilibrium models.

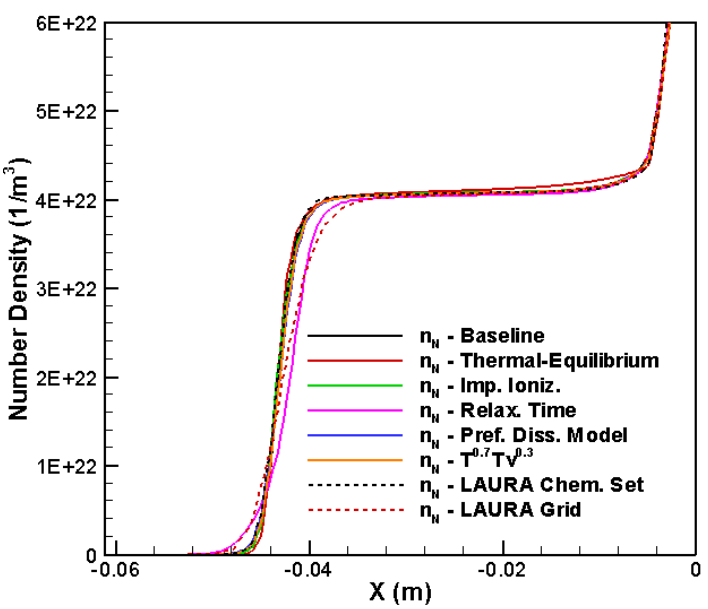

Figure 14. Atomic nitrogen number density along the stagnation line for the 1636 seconds, 11-species, noncatalytic wall case calculated using different thermal non-equilibrium models. 


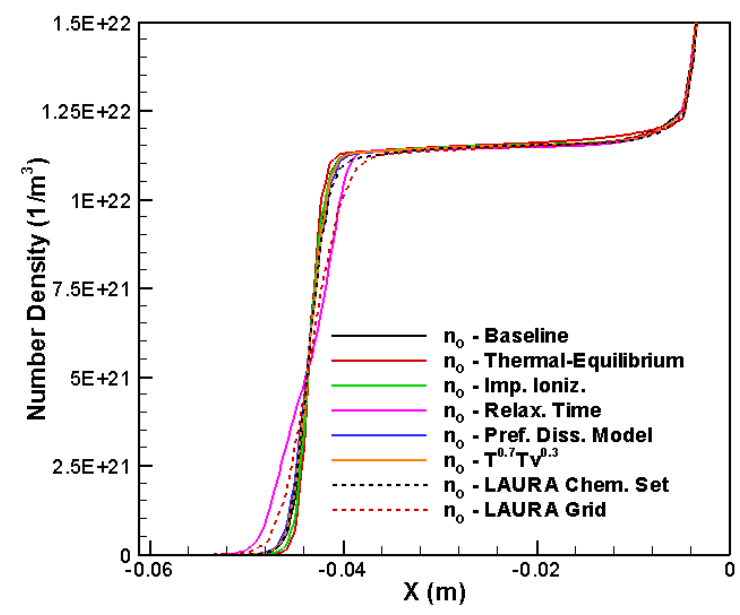

Figure 15. Atomic oxygen number density along the stagnation line for the 1636 seconds, 11-species, noncatalytic wall case calculated using different thermal non-equilibrium models.

one calculated by DPLR. This modification also reduces the maximum vibrational temperature as observed in Fig. 12. Figures 13 to 15 show that the impact on number densities of electrons, nitrogen and oxygen is negligible. The change in radiation is negligible because the change in vibrational temperature occurs before the number densities of electrons, nitrogen and oxygen are significant to the radiation calculation.

The third modification increases the translational-vibrational energy transfer relaxation time. This study aims to contrast the thermal-equilibrium case by choosing a value that is larger than the one calculated using the standard Millikan and White correlation. The value chosen is $\tau_{v}=1 \times 10^{-5}$ seconds. Figure 11 shows that this modification significantly increases the translational temperature which gets as high as $48,000 \mathrm{~K}$. This happens because the energy transfer rate between the translational and the other energy modes is slowed down. Due to the higher temperature, the density close to the shock is smaller, thus increasing the shock stand-off distance. Figure 12 shows that the maximum vibrational temperature is smaller than the one in the baseline solution. Also, the maximum vibrational temperature happens closer to the body. The steep increases at the shock in the number density profiles, shown in Figs. 13 to 15, are also closer to the body. The combined effect is a slight decrease in the calculated radiation.

The fourth modification uses a preferential dissociation model that accounts for the fact that molecules at higher energy vibrational states are more likely to dissociate. Figures 11 and 12 show that this modification does not cause significant changes in the translational or vibrational temperature profiles. Figures 13 to 15 show that there are no noticeable changes in the number density profiles either. Not surprisingly, the calculated radiation is very close to the baseline.

The fifth modification uses different parameters for calculating the temperature that is used in the forward dissociation reaction rates (Park's temperature). DPLR and LEMANS use $T_{p}=T^{0.5} \times T_{V}^{0.5}$ while LAURA uses that $T_{p}=T^{0.7} \times T_{V}^{0.3}$. This modification causes almost no difference in the translational and vibrational temperature profiles as can be seen in Figs. 11 and 12, respectivelly. Figures 13 to 15 show that there are no changes in the number density profiles. This explains the agreement between the radiation calculated in this case and in the baseline solution.

The sixth modification uses LAURA chemistry set as reported in Ref. 9. This modification does not cause significant changes in the translational nor in the vibrational temperature profiles, as can be observed in Figs. 11 and 12. However, the different chemistry set does cause a change in the electron number density profile. Figure 13 shows that this modification eliminates the peak in the electron number density that occurs close to the shock wave in the LEMANS baseline solution. The number density profiles for nitrogen and oxygen are mostly the same as in the baseline, as shown in Figs. 14 and 15. The reduction in electron number density causes a reduction on the radiation heat transfer when compared to the baseline.

The seventh and last modification uses the same grid as LAURA. This allows to investigate the diffusive character of LAURA's solution close to the shock wave. The coarser grid increases the amount of dissipation 
added at the shock wave, which broadens the translational temperature jump seen in Fig. 11. The broadening of the shock gives extra time for the energy transfer between the translational and the vibrational energy modes, thus causing a decrease in the maximum translational temperature. Figure 12 shows that the maximum vibrational temperature is a little smaller and that the steep increase in vibrational temperature happens sooner because of the thicker shock. Figures 13 to 15 show that the number density profiles are more diffused, in qualitative agreement with the LAURA profiles in Figs. 8 to 10. As a result, the calculated radiation shows better agreement with LAURA calculation.

\section{Concluding Remarks}

A code for the simulation of hypersonic flows at reentry conditions is developed. The code, called LEMANS, models the flow using the Navier-Stokes equations modified to account for thermochemical nonequilibrium and weak ionization effects. The equations are solved using a finite-volume method on unstructured grids with inviscid fluxes discretized using a Flux Vector Splitting scheme. The time march is performed implicitly using a point or line implicit method. The code is also parallelized using MPI to take advantage of parallel processing.

LEMANS is successfully used to simulate the convective heat transfer to the FIRE-II spacecraft at different trajectory points. The results are in remarkable agreement with two other established CFD codes routinely used in reentry calculations: DPLR (developed at NASA Ames) and LAURA (developed at NASA Langley). For the 1636 seconds case, LEMANS is very close to DPLR due to many similarities in the physical and numerical modeling. Overall, the differences in convective heating between LEMANS, DPLR and LAURA stay under $7 \%$ for 11-species air model calculations. LEMANS flow field results are also employed to perform stagnation point radiative heating calculations using NEQAIR. The results are in good agreement with the ones obtained using DPLR and LAURA. A sensitivity study restricted to modifications in the thermochemical non-equilibrium model is also performed. It is found that reasonable changes in some of the parameters of this model do not lead to very significant changes in the radiation heat transfer. One of the largest source of discrepancy is the computational grid. This result indicates that CFD grids used in radiation analysis need to be refined at the shock wave in addition to at the boundary layer.

\section{Acknowledgments}

The authors are indebted to the Francois-Xavier Bagnoud Foundation and the Rackham School of Graduate Studies at the University of Michigan which provide a fellowship to the first author. Additional funding is provided by the Space Vehicle Technology Institute, under NASA grant NCC3-989 with joint sponsorship from the Department of Defense. The authors also would like to thank Dr. David Hash and Dr. Mike Wright from NASA Ames Research Center and Dr. Peter Gnoffo from NASA Langley Research Center for results, grids and discussions on the flow field calculations, and Dr. Dinesh Prabhu of the Eloret Corporation for assistance in the use of NEQAIR.

\section{References}

\footnotetext{
${ }^{1}$ Gnoffo, P. A., Weilmuenster, K. J., Hamilton II, H. H., Olynick, D. R., and Venkatapathy, E., "Computational Aerothermodynamic Design Issues for Hypersonic Vehicles," Journal of Spacecraft and Rockets, Vol. 36, 1999, pp. $21-43$.

${ }^{2}$ Candler, G. V., The Computational of Weakly Ionized Flow in Nonequilibrium, Ph.D. thesis, Stanford University, California, June 1988.

${ }^{3}$ Olynick, D. R., Henline, W. D., Chambers, L. H., and Candler, G. V., "Comparison of Coupled Radiative Flow Solutions with Project Fire II Flight Data," Journal of Thermophysics and Heat Transfer, Vol. 9, 1995, pp. 586-594.

${ }^{4}$ Wright, M., Loomis, M., and Papadopoulos, P., "Aerothermal Analysis of the Project Fire II Afterbody Flow," Journal of Thermophysics and Heat Transfer, Vol. 17, 2003, pp. 240-249.

${ }^{5}$ Wright, M., Prabhu, D., and Martinez, E., "Analysis of Afterbody Heating Rates on the Apollo Command Module, Part 1: AS-2-2," AIAA Paper 2004-2456, 2004.

${ }^{6}$ Hollis, B. R., Striepe, S. A., Wright, M. J., Bose, D., Sutton, K., and Takashima, N., "Prediction of the Aerothermodynamic Environment of the Huygens Probe," AIAA Paper 2005-4816, 2005.

${ }^{7}$ Edquist, K. T., "Afterbody Heating Predictions for a Mars Science Laboratory Entry Vehicle," AIAA Paper 2005-4817, 2005.

${ }^{8}$ Bertin, J. J., Hypersonic Aerothermodynamics, AIAA Education Series, 1994.

${ }^{9}$ Hash, D., Olejniczak, J., Wright, M., Prabhu, D., Pulsonetti, M., Hollis, B., Gnoffo, P., Barnhardt, M., Nompelis, I., and
} 
Candler, G., "FIRE II Calculations for Hypersonic Nonequilibrium Aerothermodynamics Code Verification: DPLR, LAURA, and US3D," AIAA Paper 2007-0605, 2007.

${ }^{10}$ Wright, M. J., A Family of Data-Parallel Relaxation Methods for the Navier-Stokes equations, Ph.D. thesis, University of Minnesota, Minnesota, June 1997.

${ }^{11}$ Gnoffo, P. A., Gupta, R. N., and Shinn, J. L., "Conservation Equations and Physical Models for Hypersonic Air Flows in Thermal and Chemical Nonequilibrium," NASA-TP-2867, NASA Langley, Hampton, Virginia, 1989.

${ }^{12}$ Scalabrin, L. C. and Boyd, I. D., "Development of an Unstructured Navier-Stokes Solver for Hypersonic Nonquilibrium Aerothermodynamics," AIAA Paper 2005-5203, 2005.

${ }^{13}$ Scalabrin, L. C. and Boyd, I. D., "Numerical Simulation of Weakly Ionized Hypersonic Flow for Reentry Configurations," AIAA Paper 2006-3773, 2006.

${ }^{14}$ Lee, J. H., "Basic Governing Equations for the Flight Regimes of Aeroassisted Orbital Transfer Vehicles," Thermal Design of Aeroassisted Orbital Transfer Vehicles, Vol. 96, AIAA, New York, 1985, pp. 3-53.

${ }^{15}$ Park, C., Nonequilibrium Hypersonic Aerothermodynamics, John Wiley \& Sons, 1990.

${ }^{16}$ Wilke, C. R., "A Viscosity Equation for Gas Mixtures," J. of Chem. Phys., Vol. 18, 1950, pp. 517-519.

${ }^{17}$ Blottner, F. G., Johnson, M., and Ellis, M., "Chemically Reacting Viscous Flow Program for Multi-Component Gas Mixtures," Sc-rr-70-754, Sandia Laboratories, Albuquerque, New Mexico, 1971.

${ }^{18}$ Vincenti, W. G. and Kruger, C. H., Introduction to Physical Gas Dynamics, Krieger Publishing Company, 1982.

${ }^{19}$ Gupta, R. N., Yos, J. M., Thompson, R. A., and Lee, K. P., "A review of reaction rates and thermodynamic and transport properties for an 11-species air model for chemical and thermal nonequilibrium calculations to 30000 K," NASA-RP-1232, 1990.

${ }^{20}$ Wright, M. J., Bose, D., Palmer, G. E., and Levin, E., "Recommended Collision Integrals for Transport Property Computations, Part 1: Air Species," AIAA Journal, Vol. 43, No. 12, 2005, pp. 2558-2564.

${ }^{21}$ Sutton, K. and Gnoffo, P. A., "Multi-Component Diffusion with Application to Computational Aerothermodynamics," AIAA Paper 1998-2575, 1998.

${ }^{22}$ Park, C., "Review of Chemical-Kinetic Problems of Future NASA Missions, I: Earth Entries," Journal of Thermophysics and Heat Transfer, Vol. 7, 1993, pp. 385-398.

${ }^{23}$ Park, C., "On Convergence of Computation of Chemically Reacting Flows," AIAA Paper 85-0247, 1985.

${ }^{24}$ Millikan, R. C. and White, D. R., "Systematics of Vibrational Relaxation," J. of Chem. Phys., Vol. 39, 1963, pp. 32093213.

${ }^{25}$ Park, C., "Assessment of two-temperature kinetic model for ionizing air," AIAA Paper 1987-1574, 1987.

${ }^{26}$ Hirsch, C., Numerical Computation of Internal and External Flows, John Wiley \& Sons, 1991.

${ }^{27}$ Nompelis, I., Computational Study of Hypersonic Double-Cone Experiments for Code Validation, Ph.D. thesis, University of Minnesota, Minnesota, May 2004

${ }^{28}$ MacCormack, R. W. and Candler, G. V., "The Solution of the Navier-Stokes Equations Using Gauss-Seidel Line Relaxation," Computers and Fluids, Vol. 17, 1989, pp. 135-150.

${ }^{29}$ Steger, J. L. and Warming, R. F., "Flux Vector Splitting for the Inviscid Gasdynamic Equations with Applications to Finite Difference Methods," Journal of Computational Physics., Vol. 40, 1981, pp. 263-293.

${ }^{30}$ Buning, P. G. and Steger, J. L., "Solution of the Two-Dimensional Euler Equations with Generalized Coordinate Transformations Using Flux Vector Splitting," AIAA Paper 82-0971, 1982.

${ }^{31}$ Druguet, M., Candler, G. V., and Nompelis, I., "Effect of Numerics on Navier-Stokes Computations of Hypersonic Double-Cone Flows," AIAA Journal, Vol. 43, No. 3, 2005, pp. 616-623.

${ }^{32}$ Jawahar, P. and Kamath, H., "A High-Resolution Procedure for Euler and Navier-Stokes Computations on Unstructured Grids," Journal of Computational Physics, Vol. 164, 2000, pp. 165-203.

${ }^{33}$ Gnoffo, P. A., "Computational Aerothermodynamics in Aeroassist Applications," Journal of Spacecrafts and Rockets, Vol. 40, No. 3, 2003, pp. 305-312.

${ }^{34}$ Venkatakrishnan, V., "Implicit Schemes and Parallel Computing in Unstructured Grid CFD," VKI-LS-1995-02, VKI Lecture Series, 1995.

${ }^{35}$ Whiting, E. E., Park, C., Liu, Y., Arnoldand, J. O., and Paterson, J. A., "NEQAIR96 Nonequilibrium and Equilibrium Radiative Transport and Spectra Program: User's Manual," NASA-RP-1389, 1996. 